\title{
A transobturator adjustable system for male incontinence: 30-month follow-up of a multicenter study
}

\author{
Salomon Victor Romano ${ }^{1}$, Wilhelm Huebner², Flavio Trigo Rocha ${ }^{3}$, Fernando Pires Vaz ${ }^{4}$, Valter Muller ${ }^{4}$, \\ Fabio Nakamura ${ }^{5}$ \\ ${ }^{1}$ Department of Urology, Hospital Durand - Urologia, Buenos Aires, Argentina; ${ }^{2}$ Department of Urology, \\ Humanis Clinic, Korneuburg, Lower Austria, Austria; ${ }^{3}$ Department of Urology, Hospital das Clinicas, São \\ Paulo, SP Brazil; ${ }^{4}$ Department of Urology, Hospital dos Servidores, Rio de Janeiro, RJ, Brazil; ${ }^{5}$ CIEM - \\ Centro de Especialidades Médicas de Florianopolis, Florianopolis, SC, Brazil
}

\section{ABSTRACT}

Purpose: To report long-term results of the Argus T adjustable system for treatment of post-prostatectomy urinary incontinence (PPI).

Materials and Methods: From October 2007 to August 2008, 37 patients with PPI were included in a prospective, single-arm, multicenter trial of treatment with the Argus $\mathrm{T}$ adjustable system (Promedon, Argentina). Preoperative evaluation included urine culture, urethrocystoscopy, urodynamic testing, 24-h pad weight test (PWT) and quality of life questionnaires. Patients were stratified according to baseline degree of incontinence (mild-moderate or severe). Postoperative evaluation included immediate PWT, quality of life questionnaires and daily use of pads at 1, 12 and 30 months.

Results and Conclusions: One patient was lost to follow-up. At the 30-month follow-up, 24/31 patients (77\%) were dry, 3/31 (10\%) improved and 4/31 (13\%) were failures. In particular, in the mild-moderate group, $8 / 8(100 \%)$ patients were dry. In the severe group, 20/28 patients (71\%) were dry, 3/28 (11\%) improved and 5/28 (18\%) were failures. Median visual analogue scale (VAS) scores dropped from 9 (4-10) to 0.5 (0-10) and International Consultation on Incontinence Questionnaire Short Form scores from (ICIQ-SF) $19(12-21)$ to $1(0-10)$. Retrograde leak point pressure increased from 18 (5-29) to 35 (22-45) $\mathrm{cm} \mathrm{H}_{2} \mathrm{O}$ after intraoperative adjustment. Complications included immediate postoperative infection in 2/36 patients (6\%) and transient inguinal and/or perineal pain in 22/36 patients (61\%). Argus T has a long-term high success rate $(86 \%$ cure + improvement at the 30-month follow-up). Good outcomes were achieved even in severe incontinence cases and maintained for over 30 months.

\section{ARTICLE INFO}

Key words:

Suburethral Slings; Prostatectomy; Urinary Incontinence; Urodynamics

Int Braz J Urol. 2014; 40: 781-9

Submitted for publication:

October 19, 2013

Accepted after revision:

March 03, 2014

\section{INTRODUCTION}

Although rare, stress urinary incontinence secondary to prostate surgery, whether for prostate cancer or benign prostatic hyperplasia (BPH), causes significant deterioration of patients' quality of life. Persistent urinary incontinence occurs in $5-10 \%$ of post radical prostatectomy patients and in $0.5-3 \%$ of post BPH surgery patients. In both situations, incontinence can be severe enough to require surgical management.

Conservative management is generally recommended during the first 6-12 months after prostatectomy. Behavioral modifications, pelvic floor muscle training and drug therapy have been the most frequently recommended options. 
Surgical interventions are the next treatment option for persistent UI. Peri-urethral injection for temporary relief, minimally invasive compression devices, fixed and adjustable slings and artificial urinary sphincters (AUS) are the current recommended forms of surgical treatment (1-3).

Suprapubic slings had seldom been used prior to Schaeffer et al. report in the late 1990s of a success rate of 75\% (cure + improvement) for a bulbourethral device in a group of 64 patients with a 2-year follow-up (4). After this initial report, several other researchers assessed this procedure and added modifications to the original design. Romano et al. (2) reported a success rate of $83 \%$ (73\% cure $+10 \%$ improved) for an adjustable bulbourethral device inserted via a suprapubic approach in a group of 48 patients with a mean follow-up of 7.5 months. The same group later reported long-term stability with a $78.8 \%$ success rate $(66.0 \%$ cure $+12.8 \%$ improved) after a mean follow-up of 45 months (5).

To find a simpler and safer approach for implantation, we evaluated a transobturator approach in 2003 (6). After proving its feasibility in 2007, we began a multicenter trial. Data from this study are reported in this paper.

\section{MATERIALS AND METHODS}

Potential subjects were screened for a prospective, single-arm, multicenter trial from October 2007 to August 2008. The study protocol was approved by the corresponding independent ethics committees and written informed consents were obtained before patients' inclusion. A group of 37 patients who met the eligibility criteria were finally selected. Inclusion criteria were 1 year or more of PPI (of any degree of severity) that had altered quality of life to the extent that the patient agreed to a surgical procedure and urodynamic confirmation of stress incontinence. Exclusion criteria included untreated urinary infection, urethral stricture, low bladder capacity (less than $200 \mathrm{~mL}$ ) and bladder stone unable to be resolved during a sling procedure.

Preoperative evaluation included a complete urologic exam, urine culture, urethrocystoscopy, 24-h PWT and quality of life questionnaires
(ICIQ-SF and VAS). Urodynamic testing was also performed to assess the filling variables of sensitivity, capacity, compliance, detrusor overactivity and to confirm the stress nature of the UI as well as the retrograde leak point pressure (RLPP) $(7,8)$. The emptying variables of free flow and voluntary detrusor contractility were also recorded. The baseline characteristics of the enrolled patients are shown in Table-1.

Patients were grouped according to their degree of incontinence into mild-moderate and severe categories. This stratification was based on 24-h PWT preoperative measurements. The patient was assessed as having mild-moderate incontinence if the leakage was less than or equal to $400 \mathrm{~g}$ and severe incontinence if it was greater than 400g (9) (Table-1).

An amendment was made to the protocol in four of the five initial centers to include a follow-up at 30 months. For this reason, 31 patients were followed at 1,12 and 30 months and the remaining five patients only at 1 and 12 months postoperatively.

Argus T adjustable systems (Promedon, Argentina) were surgically implanted in the patients. This system consists of two cone columns that serve as fixation arms and a central pad made of radiopaque silicone foam. The system is completed by placing two rings (washers) on each fixation arm to provide safe anchoring and positioning of the device against the fibro muscular tissue of the obturator foramen.

The surgical procedure is as follows. The patient is placed in the lithotomy position, under spinal or general anesthesia. As has previously been described $(2,5,10)$, a perineal incision is performed in the same way as for suprapubic or transobturatory devices to dissect the bulbar urethra at the level of the inter bulbospongiosus and ischiocavernous muscles area. The bulbospongiosus muscles are left in situ and the urethra is not mobilized from the central tendon. A helical needle is then introduced via the inguinal fold, $2 \mathrm{~cm}$ below the insertion of the adductor magnus muscle, using an outside-in approach such that the needle tip appears in the dissected area of the perineum. The fixation arm is pulled out along the needle path and the procedure repeated on the other 
Table 1 - Patients' baseline characteristics.

\begin{tabular}{|c|c|c|}
\hline Characteristic & $\mathrm{N}(\%)$ & Median (range) \\
\hline Age (years) & & $70(58-81)$ \\
\hline $\operatorname{RLPP}\left(\mathrm{cmH}_{2} \mathrm{O}\right)$ & & $18(5-29)$ \\
\hline \multicolumn{3}{|l|}{ Underlying pathology } \\
\hline Post-prostate cancer & $30(81)$ & \\
\hline Post-adenomectomy & $7(19)$ & \\
\hline Adjuvant Radiotherapy & $2(5)$ & \\
\hline Prior incontinence surgery & $6(16)$ & \\
\hline Argus (retropubic) & $1(3)$ & \\
\hline ProACTТM (parurethral balloon) & $3(8)$ & \\
\hline Macroplastique ${ }^{T M}$ (injectable bulking agent) & $2(5)$ & \\
\hline Degree of incontinence & & 24-h PWT (g) \\
\hline Mild-moderate & $8(22)$ & $215(100-350)$ \\
\hline Severe & $29(78)$ & $1200(500-2880)$ \\
\hline Total & $37(100)$ & $1100(100-2880)$ \\
\hline
\end{tabular}

side. After placing the rings on the fixation arms and checking that the foam pad is centered, symmetrical adjustment is performed one cone at a time until an $\mathrm{RLPP}$ of 30 to $40 \mathrm{cmH}_{2} \mathrm{O}$ is achieved. The ruler (included in the kit) must be positioned with the 0 (zero) at the level of the patient's pubis (Figures 1-4).

The urethral catheter is left in place for 24 to $48 \mathrm{~h}$. Patients are given intraoperative cephalosporin $1 \mathrm{~g}$ and gentamicin $80 \mathrm{mg}$ every $12 \mathrm{~h}$ until the cathe- ter has been removed, after which oral ciprofloxacin (500mg every 12h) is prescribed for 7-10 days.

The follow-up plan included evaluations at 1 , 12 and 30 months. Quality of life was assessed by the ICIQ-SF questionnaire and a VAS scale (from 0 [no discomfort] to 10 [very uncomfortable]). The degree of incontinence was objectively assessed on the basis of 24-h PWT at the first postoperative follow-up visit (1 month) and by daily pad use on the other visits.

Figure 1 - ARGUS T adjustable system - Promedon SA.
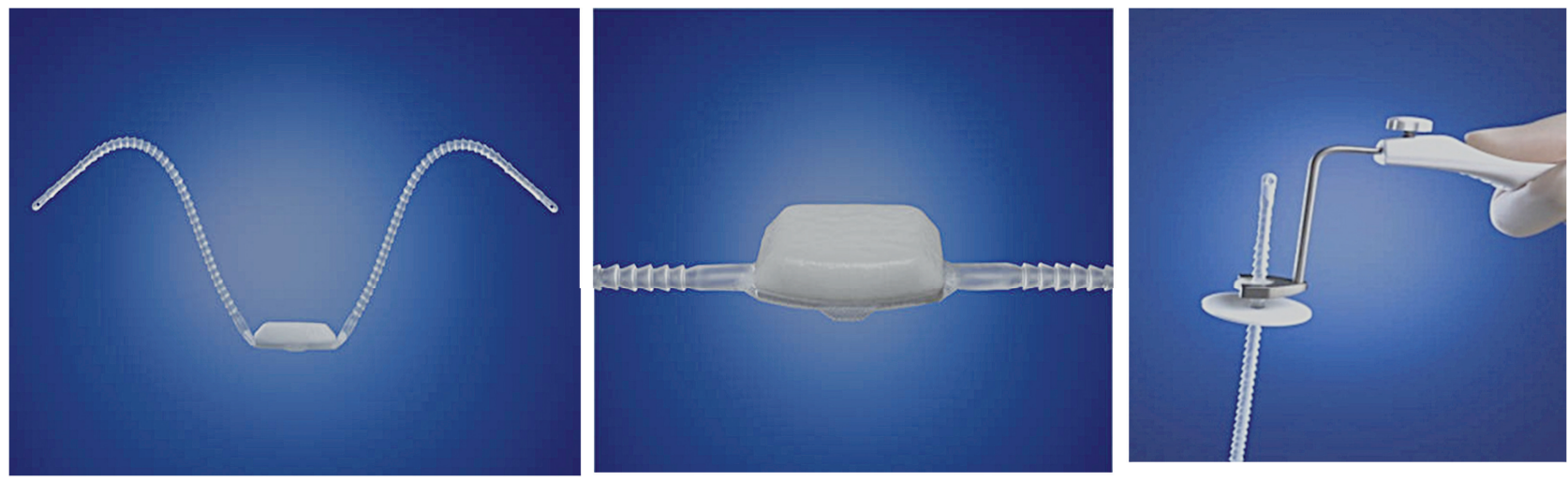
Figure 2 - Left - tip of left needle in the perineal wound. Right - Implant: Pad, Rings and hidden fixation arms, in final position after the RLPP measure.
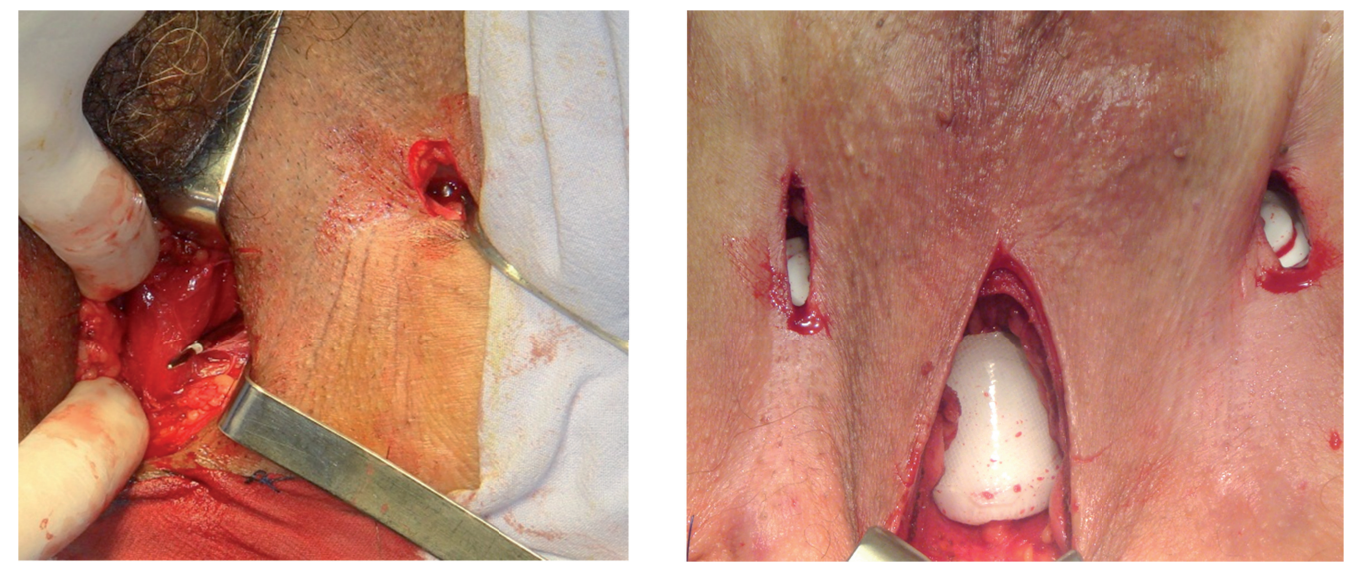

Figure 3 - Left - urethral lumen before the procedure. Right - urethral lumen after RLPP adjust.
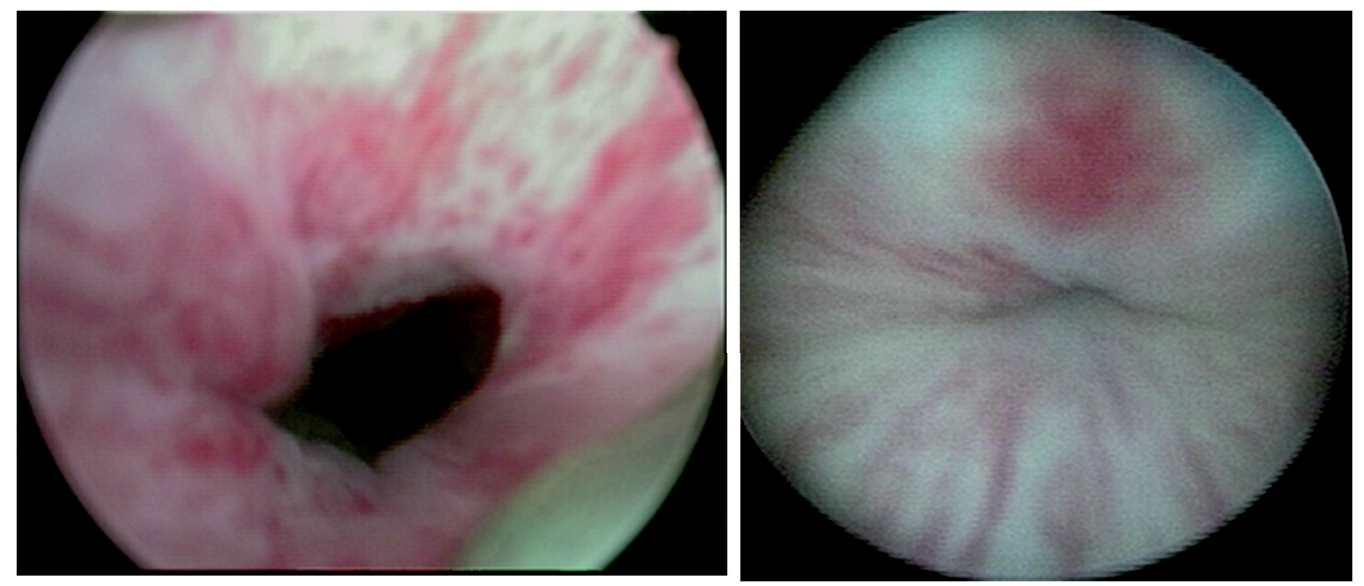

Figure 4 - Left - final position of the implant in a plain x-ray. Right - final position of the implant in a MRI 3D reconstruction.
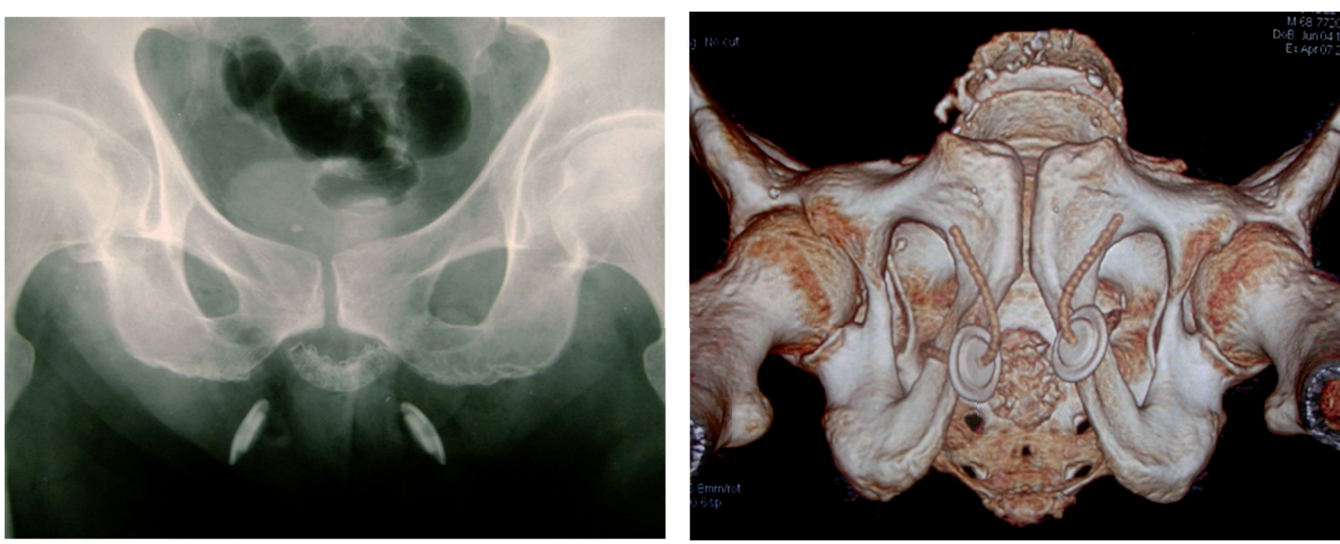
The patients' statuses were classified according to the daily use of pads as dry (no pads or one for protection), improved (one wet pad a day) or failure (two or more wet pads daily or implant removal).

During follow-up, adverse events were also recorded and the Clavien-Dindo Classification of Surgical Complications was used to report them $(11,12)$.

\section{RESULTS}

One of the 37 study patients was lost to follow-up after the first postoperative visit. As has already been stated, five patients were only followed up for 12-months. The study subject distribution tree is shown in Figure-5.

Figure 5 - Study subjects' distribution tree.

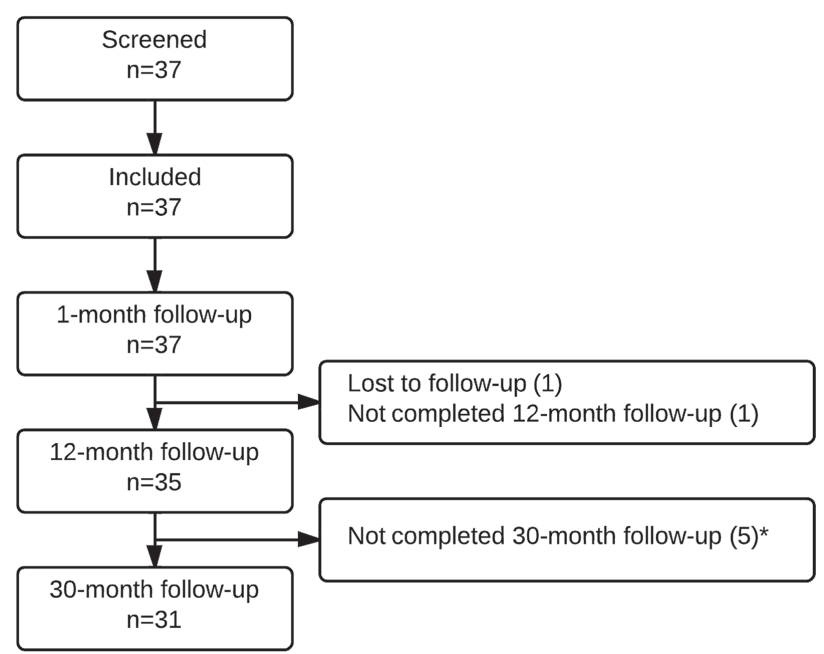

${ }^{*}$ The five patients who did not complete the 30-month follow-up were from the center that did not amend the protocol to extend the duration of follow-up.
In the short term, at the 1-month follow-up the median 24-h PWT had improved from $1100 \mathrm{~g}(100-2880 \mathrm{~g})$ overall to $\mathrm{Og}(0-35 \mathrm{~g})$ in dry patients and 50g (50-72g) in improved patients. The RLPP measured during the process of surgical adjustment of the implant increased from 17 (5-29) to $35(22-45) \mathrm{cmH}_{2} \mathrm{O}$.

All quality of life indicators changed favorably: the median VAS score from $9(4-10)$ to 0.5 $(0-10)$ and the ICIQ-SF from $19(12-21)$ to $1(0-10)$ (Table-2).

In the mild-moderate group, all eight patients achieved continence. In the severe group 20/28 patients achieved continence and 3/28 patients improved, requiring only one pad per day (Table-3).

No one in the mild-moderate group needed postoperative readjustment because they all achieved continence postoperatively. In the severe group, seven patients (25\%) who remained incontinent after surgery gave their consent to readjustment. The median time from the initial surgery to readjustment was 14 (7-25) months. Results of readjustment are reported in Table- 4 .

Immediate infection occurred in 2/36 patients (5.6\%). One of them required implant removal (Grade III-a). This patient had previously had a ProACT ${ }^{\mathrm{TM}}$ (Uromedica, USA) implanted; this had also required removal because of infection. The other patient with immediate infection was treated with local wound care and antibiotics and required no further intervention after 3 months (Grade II). Upon catheter removal, postoperative urinary retention occurred in 2/36 patients (5.6\%). In one of these patients, the retention was overcome by postoperative readjustment (loosening) of

Table 2 - Postoperative results.

\begin{tabular}{lccccc}
\hline & & Pre-op & 1 month & 12 months & 30 months \\
\hline Daily pad use N (\%) & Dry & & $26(70)$ & $28(80)$ & $24(77)$ \\
& Improved & & $6(16)$ & $3(9)$ & $3(10)$ \\
& Failed & & $5(14)$ & $4(11)$ & $4(13)$ \\
ICIQ-SF score median (range) & & $19(12-21)$ & $2(0-20)$ & $2(0-20)$ & $1(0-10)$ \\
VAS score median (range) & & $9(4-10)$ & $1(0-10)$ & $0(0-10)$ & $0.5(0-10)$ \\
\hline
\end{tabular}


Table 3 - Results segregated by baseline degree of incontinence, according to each patient's last follow-up.

\begin{tabular}{lcc}
\hline Baseline degree of incontinence & \multicolumn{2}{c}{ Postoperative outcome } \\
\hline Mild-moderate & Dry & $8 / 8(100 \%)$ \\
& Improved & $0 / 8(0 \%)$ \\
Severe & Failed & $0 / 8(0 \%)$ \\
& Dry & $20 / 28(71 \%)$ \\
& Improved & $3 / 28(11 \%)$ \\
& Failed & $5 / 28(18 \%)$ \\
\hline
\end{tabular}

Table 4 - Readjustment of the Argus T device postoperatively.

\begin{tabular}{lccc}
\hline Patient group & Number of readjustments & \multicolumn{2}{c}{ Outcome after readjustment } \\
\hline Mild-moderate & $0 / 8(0 \%)$ & \multicolumn{2}{c}{ NA } \\
Severe & $7 / 28(25 \%)$ & Dry & $5 / 7(72 \%)$ \\
& & Improved & $1 / 7(14 \%)$ \\
& & Failed & $1 / 7(14 \%)$ \\
\hline
\end{tabular}

the implant, thus decreasing the RLPP (Grade III-a). The other patient had impaired bladder contractions (hypocontractility) postoperatively: after 6 months of intermittent catheterization, he regained spontaneous bladder evacuation with no post-voiding residual urine (Grade II).

Transient inguinal and/or perineal pain was reported immediately after surgery by $22 / 37$ patients (61\%). The pain resolved within 3-4 weeks after treatment with analgesics, nonsteroidal anti-inflammatory drugs (Grade I) and/or corticosteroids (Grade II). In one patient (2.8\%), pain persisted for 2 months before resolution.

\section{DISCUSSION}

As recently as 10 years ago, the only reliable surgical treatment for PPI was the AUS (13, 14). Although it has been associated with significant complications such as infection, erosion and mechanical failure, including urethral atrophy with recurrent incontinence, and a revision rate of greater than $50 \%(15,16)$, this procedure continues to play a predominant role.
During the last decade, a series of adjustable and non-adjustable devices have been developed for treating PPI. All of them attempt to achieve continence by urethral coaptation, rather than by closing the urethral lumen as the AUS does. The latter works as a hydraulically operated open-close valve that produces either complete obstruction of the urethral lumen or complete opening, whereas the coaptation devices aim to increase the baseline RLPP to reinforce the sphincteric mechanism. Therefore, with coaptation devices continence and micturition are ruled by the normal physiological balance between intravesical pressure and urethral resistance. Adjustable devices offer a great advantage over the non-adjustable ones: their ability to be adapted to changes in patients' conditions. Examples of adaptable devices are the Argus (Promedon, Argentina), Remeex (Neomedic, Spain), ATOMS (A.M.I., Austria) and ProACT ${ }^{\mathrm{TM}}$ (Uromedica, USA) devices (5, 17-20).

Among the non-adjustable devices are various models of autologous facials slings or polypropylene tapes such as Invance ${ }^{\mathrm{TM}}$ (bone anchored sling), AdVance ${ }^{\mathrm{TM}}$ and other devices (21-27). 
The AdVance ${ }^{\mathrm{TM}}$, one of the most commonly used non-adjustable devices, reportedly has very good results (around $80 \%$ ) in selected cases of mild to moderate PPI (3). It is claimed that this retro-urethral transobturatory sling works by repositioning the residual sphincter to an intra-abdominal location by mobilizing the urethra from the perineal central tendon, thus avoiding the urethral hypermobility that can be caused by surgery. At the same time, the urethra is occluded by the sling, which should be implanted under tensioning, as is recommended $(25,26)$.

The main advantage of adjustable syste$\mathrm{ms}$ is that they allow postoperative increasing or decreasing of sling tension to improve or correct initial outcomes. Sling tension can be increased to augment urethral resistance in patients whose incontinence persists. In addition, sling tension can be decreased by loosening the sling, thus decreasing the RLPP, in patients with urinary retention or obstruction. In this study, five patients who remained incontinent after surgery benefited from this advantage. They achieved continence (dry patients) after postoperative sling adjustments. Furthermore, by loosening the urethral coaptation postoperatively, it was possible to reverse the urinary retention of the patient who had not been able to reverse it naturally. Argus T's adjustment rings provide reliable fixation, which helps to maintain the coaptation achieved during surgery. These rings also offer a point of reference for postoperative tension adjustments.

Soljanik et al. (28) highlighted the need for reducing sling slippage and failure at short-term follow-up in patients with the AdVance ${ }^{\mathrm{TM}}$ device. They improved the surgical technique by tunneling the sling arms subcutaneously and using at least four non-absorbable sutures instead of an absorbable one.

It is important to note that, in most patients, the ability to adjust the Argus T to an intraoperative RLPP of 30 to $40 \mathrm{cmH}_{2} \mathrm{O}$, combined with the reliable anchoring supported by the rings, resulted in sustained positive outcomes without the need to perform subsequent adjustments. The recommended RLPP range has been established over more than 10 years of experience, with the aim of applying the minimum pressure in the urethra necessary to achieve continence while minimizing pain, erosion and obstruction $(2,5,29)$.

In the mild-moderate incontinence patients, who comprised $22 \%$ of study patients, the results obtained were excellent (100\% continence). In addition, the patients with severe incontinence (78\% of study patients) achieved a success rate of $82 \%$ (71\% cure $+11 \%$ improvement). These figures are encouraging in terms of efficacy and, remarkably, the results are good regardless of the severity of incontinence preoperatively. Some devices that have shown promising results in patients with mild to moderate incontinence, such as the AdVance $^{\mathrm{TM}}$, are relatively ineffective in, and therefore not indicated for, patients with severe incontinence $(3,30)$. Consequently, in severe cases for which options for effective surgical treatment are limited, the Argus $\mathrm{T}$ is an attractive alternative, as evidenced by the results presented in this paper.

Infections occurred in our series only during the immediate postoperative period and are therefore seemingly related to intraoperative contamination. However, our infection rates are similar to or even lower than those reported for other implants used for management of male incontinence, such as the AUS and Argus devices, both of which are also made from silicone $(5,16)$.

In summary, historically bulbourethral compression devices have been a prominent component of the armamentarium for treatment of PPI and have achieved very satisfactory results in 65 to $90 \%$ of patients $(3,5,10,17,21,22,24,26,27)$. Based on examination of data from medium and long-term follow-up, we believe that the common goal of all these devices is to create mild bulbourethral pressure that allows coaptation of the mucosa, thus controlling incontinence. Indeed, it appears that the lower the passive resistance required to achieve continence, the greater the likelihood of avoiding dysuria, urinary retention, pain and erosion (29).

\section{CONCLUSIONS}

We found that the Argus T adjustable system can be easily and safely implanted through a transobturator approach, providing a high success rate (86\% cure + improvement). We achieved good 
outcomes even in patients with severe incontinence and these were sustained during 30 months of follow-up. This device is a valuable treatment option for most patients with PPI.

\section{CONFLICT OF INTEREST}

None declared.

\section{REFERENCES}

1. Secin FP, Martínez-Salamanca JI, Eilber KS. Limited efficacy of permanent injectable agents in the treatment of stress urinary incontinence after radical prostatectomy. Arch Esp Urol. 2005; 58: 431-6.

2. Romano SV, Metrebian SE, Vaz F, Muller V, D'Ancona CA, Costa DE Souza EA, et al. An adjustable male sling for treating urinary incontinence after prostatectomy: a phase III multicentre trial. BJU Int. 2006; 97: 533-9.

3. Welk BK, Herschorn S. The male sling for post-prostatectomy urinary incontinence: a review of contemporary sling designs and outcomes. BJU Int. 2012; 109: 328-44.

4. Schaeffer AJ, Clemens JQ, Ferrari M, Stamey TA. The male bulbourethral sling procedure for post-radical prostatectomy incontinence. J Urol. 1998; 159: 1510-5. Erratum in: J Urol 1998;160:136.

5. Romano SV, Metrebian SE, Vaz F, Muller V, D'Ancona CA, de Souza EA, et al. Long-term results of a phase III multicentre trial of the adjustable male sling for treating urinary incontinence after prostatectomy: minimum 3 years. Actas Urol Esp. 2009; 33: 309-14.

6. Palma PC, Dambros M, Thiel M, Romano V, Griguol O, Riccetto CL, et al. Readjustable transobturator sling: a novel sling procedure for male urinary incontinence. Urol Int. 2004; 73: 354-6.

7. Comiter CV, Sullivan MP, Yalla SV. Retrograde leak point pressure for evaluating postradical prostatectomy incontinence. Urology. 1997; 49: 231-6.

8. Comiter CV, Nitti V, Elliot C, Rhee E. A new quadratic sling for male stress incontinence: retrograde leak point pressure as a measure of urethral resistance. J Urol. 2012; 187: 563-8.

9. Kumar A, Litt ER, Ballert KN, Nitti VW. Artificial urinary sphincter versus male sling for post-prostatectomy incontinence--what do patients choose? J Urol. 2009; 181: 1231-5.

10. Romano SV, Hübner WA, Trigo Rocha $F$, Muller $V$ and Nakamura F. Argus $T$ for post prostatectomy urinary incontinence - Outcomes minimum 12 months of the multicentre trial. Neurourol. Urodyn, 2010; 29: 918-9.
11. Dindo D, Demartines N, Clavien PA. Classification of surgical complications: a new proposal with evaluation in a cohort of 6336 patients and results of a survey. Ann Surg. 2004; 240: 205-13.

12. Clavien PA, Barkun J, de Oliveira ML, Vauthey JN, Dindo D, Schulick RD, et al. The Clavien-Dindo classification of surgical complications: five-year experience. Ann Surg. 2009; 250: 187-96.

13. Herschorn S, Bruschini H, Comiter C, Grise P, Hanus T, Kirschner-Hermanns R, et al. Surgical treatment of stress incontinence in men. Neurourol Urodyn. 2010; 29: $179-90$.

14. Bauer RM, Gozzi C, Hübner W, Nitti VW, Novara G, Peterson $A$, et al. Contemporary management of postprostatectomy incontinence. Eur Urol. 2011; 59: 985-96.

15. Tse V, Stone AR: Incontinence after prostatectomy: the artificial urinary sphincter. BJU Int. 2003; 92: 886-9.

16. Wang R, McGuire EJ, He C, Faerber GJ, Latini JM. Long-term outcomes after primary failures of artificial urinary sphincter implantation. Urology. 2012; 79: 922-8.

17. Sousa-Escandón A, Rodríguez Gómez JI, Uribarri González C, Marqués-Queimadelos A. Externally readjustable sling for treatment of male stress urinary incontinence: points of technique and preliminary results. J Endourol. 2004; 18: 113-8.

18. Hübner WA, Schlarp OM. Treatment of incontinence after prostatectomy using a new minimally invasive device: adjustable continence therapy. BJU Int. 2005; 96: 587-94.

19. Trigo-Rocha F, Gomes CM, Pompeo AC, Lucon AM, Arap S. Prospective study evaluating efficacy and safety of Adjustable Continence Therapy (ProACT) for post radical prostatectomy urinary incontinence. Urology. 2006;67:965-9.

20. Seweryn J, Bauer W, Ponholzer A, Schramek P. Initial experience and results with a new adjustable transobturator male system for the treatment of stress urinary incontinence. J Urol. 2012; 187: 956-61.

21. Stern JA, Clemens JQ, Tiplitsky SI, Matschke HM, Jain PM, Schaeffer AJ. Long-term results of the bulbourethral sling procedure. J Urol. 2005; 173: 1654-6.

22. Athanasopoulos A, McGuire EJ. Efficacy of the bulbourethral autologous sling in treating male stress urinary incontinence: a three-year experience from a single center. Int Urol Nephrol. 2010; 42: 921-7.

23. Madjar S, Jacoby K, Giberti C, Wald M, Halachmi S, Issaq $\mathrm{E}$, et al. Bone anchored sling for the treatment of postprostatectomy incontinence. J Urol. 2001; 165: 72-6.

24. Samli M, Singla AK. Absorbable versus nonabsorbable graft: outcome of bone anchored male sling for post-radical prostatectomy incontinence. J Urol. 2005; 173: 499-502.

25. Rehder P, Gozzi C. Transobturator sling suspension for male urinary incontinence including post-radical prostatectomy. Eur Urol. 2007; 52: 860-6. 
26. Rehder $\mathrm{P}$, Mitterberger MJ, Pichler R, Kerschbaumer A, Glodny B. The 1 year outcome of the transobturator retroluminal repositioning sling in the treatment of male stress urinary incontinence. BJU Int. 2010; 106: 1668-72.

27. John $H$. Bulbourethral composite suspension: a new operative technique for post-prostatectomy incontinence. J Urol. 2004; 171: 1866-70; discussion 1869-70.

28. Soljanik I, Gozzi C, Becker AJ, Stief CG, Bauer RM. Risk factors of treatment failure after retrourethral transobturator male sling. World J Urol. 2012; 30: 201-6.
29. Hübner WA, Gallistl H, Rutkowski M, Huber ER. Adjustable bulbourethral male sling: experience after 101 cases of moderate-to-severe male stress urinary incontinence. BJU Int. 2011; 107: 777-82.

30. Rehder P, Freiin von Gleissenthall G, Pichler R, Glodny B. The treatment of postprostatectomy incontinence with the retroluminal transobturator repositioning sling (Advance): lessons learnt from accumulative experience. Arch Esp Urol. 2009; 62: 860-70.

Correspondence address:

Salomon Victor Romano, MD Hospital Durand - Urologia Diaz Velez 5044

Buenos Aires 1405, Argentina E-mail: sromano1@arnet.com.ar 